\title{
A PHILOSOPHY OF THE EARTH AND UNIVERSE
}

The Robert Mackenzie Johnston Lecture to the Royal Society of Tasmania

May 2nd, 1977

\section{by S. Warren Carey}

\section{ABSTRACT}

The universal null emerges with simplicity as an inevitable consequence of many physical phenomena and principles.

\section{RETROSPECT}

My first address to this Society was thirty-two years ago in this hall, on Tasmania's place in the geological structure of the world. What I said then was contrary to the beliefs of the overwhelming majority of the geologists of the time. Today it is the commonly accepted dogma throughout the world. But during the intervening decades I have added to that concept of continental dispersion the still more radical proposition of accelerating expansion of the earth. So once again I am the odd man out.

Like Kipling's cat that walked by itself, the way of the loner is much harsher than the smoothed path of those who walk in the mutual admiration society of the orthodox. Bondi (1960 p.6) has pointed out that whereas we should seek economy of hypotheses, there is nevertheless a widespread tendency not to count assumptions implicit in the currently accepted doctrines, and so to excise with Occan's razor a new alternative which involves no more assumptions than the popular concept. Unfortunately, scientists being human, much more severe demands and il1-founded rebuttals, even slurs ad hominem, are approvingly thrown at the unorthodox loner.

Disheartening as it has been to have one's best work rejected by the academies on such grounds, nevertheless, the painful process has in the long run been beneficial, like the torturous forging and tempering of steel. For throughout the decades I have been forced to dig deeper and deeper into fundamental assumptions and "axioms", first of geology, then of all science, to the ultimate source. From this quest has slowly emerged a new philosophy of the universe, which is the subject of my address to-night.

Before delving into this ultimate subject, first I should tell you briefly why I am convinced that the earth is expanding, when so many of my colleagues think different1y. For it was my need to explain the dispersion of the continents which led me to earth expansion, and my need to explain earth expansion, and that ancient rocks did not demand substantially greater gravity acceleration, which forced me to examine the assumptions of cosmology, and thence, the origin of the universe.

\section{THE EXPANDING EARTH}

The majority of all geologists and geophysicists now agree that all continents have separated from each other and that the existing floors of all oceans have developed during the last twentieth of the earth's life. Most geologists assume that the radius of the earth is virtually constant, and therefore that, as new ocean floors have developed between the continents, so equal areas of ocean floors have been swallowed and removed. The ocean trenches which girdle the Pacific have been identified as the sites of crustal consumption. 
Up to a quarter of a century ago, I too made these assumptions; until it became increasingly clear that this explanation led to fatal paradoxes when applied to the whole earth, and that earth expansion at an accelerating rate was inescapable.

In general, all evidence indicating dispersion of the continents and growth of new oceanic crust is satisfied equally by the constant-radius and earth-expansion models. But several other major facts, which I have set out more fully elsewhere (Carey 1976), deny the constant-radius model and imply large expansion:

(1) Palaeomagnetic and palaeontological data agree that North America is more than $30^{\circ}$ nearer the north pole than it was in the Permian Period. Likewise Europe is some $40^{\circ}$ further north, and Siberia about $17^{\circ}$ further north. Hence all continents have converged on the Arctic by large amounts since the Permian. Yet the Arctic has been a region of extension since that time, with no subduction between these converging blocks. This is impossible except on an expanding earth. Data from the Triassic, Jurassic, and Cretaceous Periods all independently give the same conclusion, but in progressively diminishing degree, indicating that the expansion has been continuous.

(2) Palaeomagnetic poles plotted from individual regions overshoot their mean pole by increasing amounts with increasing age back into the Tertiary, which implies that a degree of arc corresponded to progressively less kilometres in earlier times. Earth expansion would produce this result.

(3) The Pacific Ocean is roughly circular and significantly less than a hemisphere. All agree that all the other oceans-Arctic, Atlantic, Indian, and the marginal seas of the Pacific--have developed since the Palaeozoic by separation of adjacent continents. Hence the modern Pacific must be only half its Palaeozoic size.

But each of the continents around the Pacific perimeter have separated from each other by large amounts during the dispersion of Pangaea. Hence we have the paradox of the Pacific Ocean reducing to half its size while its perimeter is substantially increasing. This is only posible on an expanding earth.

(4) All reconstructions of Palaeozoic Pangaea show a gap tapering from the perimeter inwards. The most commonly favoured reconstruction shows this gap between Australia and southeast Asia, diminishing westwards along the Tethys to near zero in the western Mediterranean. Yet palaeogeography right through the Palaeozoic requires a close connection down the east coast of Asia to Australia and New Zealand. This paradox results from the assumption of a constant-radius earth, and evaporates on a reconstruction with smaller radius.

Likewise India has close palaeogeographic ties with Australia, Antarctica, Madagascar, East Africa, Arabia, and also with Persia and Afghanistan. Authors variously hold some of these, and sacrifice the others. All cannot be satisfied concurrently except on an expanding earth. Similarly palaeogeography requires Malaysia to have been closely tied to Gondwanaland, yet McElhinny, Haile and Crawford (1974) conclude from their palaeomagnetic data that Malaysia could not have been part of Gondwanaland. What they should have concluded is that Malaysia could not have been part of Gondwanaland if the present earth radius be assumed for the past.

(5) The subduction mode1 requires both Africa and Antarctica to have swallowed within continental Africa and continental Antarctica respectively an area of ocean floor equal to the area of these continents, and these subduction zones are required to be currently active. Such subduction zones do not exist. The plate-subduction model cannot be applied globally.

(6) According to North American data the Triassic equator passed through Texas 


\section{S. Warren Carey}

and Florida. But according to South American data the Triassic equator passed through northern Colombia and Venezuela. The constant-radius model requires subduction and crustal elimination latitudinally through the Greater Antilles. This would result in missing palaeolatitudes between North and South America. The fact is that palaeolatitudes are duplicated, which is what is required by earth expansion. The same double-equator paradox occurs between Africa and Europe, and between Australia and Asia.

These and several other facts led me a quarter of a century ago to progress from the dispersion-subduction mode1 to the dispersion-expansion model.

Critics demanded of me a physical explanation of the cause of such gross expansion before they would take it seriously, even though their own model lacked adequate physical base. Many causes have been suggested to explain expansion. Paramorphic phase changes in the deep interior during the accelerating outgassing of the earth goes part of the way, and so does the progressive decline of the gravitational constant (as deduced by Dirac on philosophical grounds, van Flandern from the recession of the moon, Brans-Dicke-Jordan from their mathematical models, and Hoyle-and-Narlikar and Wesson from cosmogony). Al1 this led me back to the most fundamental enigma of all, that had troubled me throughout my life--the origin and evolution of the universe itse1f.

\section{THE ENIGMATIC UNIVERSE}

Since the dawn of pre-history everyone who has contemplated the stars has wondered how, whence, when, why, and whither? Most have shunted the problem into the too-hard basket. Their savants, denied such escape, invoked multiple gods, single gods, or pantheism. Most assumed a special initial state - the Egyptian egg, the Hindu tortoise bearing the elephants which supported the world, the Polynesian air god Tangaloa, or the pregnant chaos of Milton. Gods of two kinds emerged: the meddling god of the Zoroastrians and of Plato, who interferes as and when he chooses, and the aloof god (as of Descartes) who created matter and motion, then left the universe to develop independently from this seed. Democritus of the fifth century B.C. was the first on record to conceive a self-created universe from the random concurrence of atoms ( - but whence his atoms?). Thence to the modern Russian materialists who dismissed all theology as superstition we should have outgrown.

But the fundamental enigma of the origin and destiny of the universe remained. Who is competent to attempt to answer? A cosmologist? Astrophysicist? Mathematician? Biologist? Theologian? Perhaps even a geologist? Alexis Carroll in his Man the Unknown wrote that to an anatomist, man is a system of bones and muscles, to a physiologist he is a sack of organs, to a biochemist an interplay of enzymes and chemical complexes, to a psychologist a mind, and to a priest, a soul. Man is all of these, and more. Each sees but an abstraction from the reality. So with the universe. Each of us filters it through our own slit, and each of us is part of it, short-spanned in space and time. We may each offer our bit to the conception of the seemingly infinite whole, and each of us must shield each other from the narvete of our narrow vision.

As a high school boy I used to wonder why was the sky black, when if the universe was infinite every line should eventually meet a star; and why did everything in the universe rotate? I was also puzzled about the claim that both momentum and energy had to be conserved. Truly, one was a vector and the other a scalar, and one essentially dynamics and the other essentially statics; but one had the form $m v$ and the other $m v^{2}$, so that, notwithstanding that sufficient independent equations could resolve such difficulties, I suspected that conditions might arise where one conservation law had to yield. It seemed clear that in such circumstances the monentum 1 aw was the more fundamental; for whereas energy may transform into many modes (mechanical, thermal, 


\section{A Phijosophy of the Earth and Universe}

electrical, elastic, magnetic, electromagnetic, and even into matter), momentum has no transformations, and momentum must be conserved as momentum still. Perhaps this was a clue to a fundamental truth of the universe-that the sum of the energy and the sum of the momentum of the universe are each zero. For at zero differing exponents become irrelevant.

Milne's Dimensionless Hypothesis

Another clue pointing in the same direction was Milne's dimensionless hypothesis (see Walker, 1936, p.121) which held that cosmological theory should have no constants with dimensions. If accepted, this seemed to imply that all physical dimensions $(M, L, T$, ) should mutually cancel at the beginning, and at all subsequent times.

\section{The Ultra Embryo}

Hubble's demonstration that nebulae were receding with velocities which increased the further they were from us implied (to quote Dirac, 1937) that "the universe had a beginning about $2 \times 10^{9}$ years ago when all the spiral nebulae were shot out from a small region of space, or perhaps from a point." What a massive point: I found this ultraheavy egg indigestible. Black holes, so dense that even radiation cannot escape, would be light-weight thistledown compared with this initial embryo. Lemaltre (1951) called it the primeval atom. Gamow (1952) called it yzem and exploded it with his initial "big bang". But surely nothing could escape such a mass concentration. This enigma again trailed the clue. Namely that matter had grown as it spread, and the regression Denken-Empemiment should have included diminishing mass, so that the initial concentration, far from the stupendous mass contemplated by Hubble and Gamow, had negligible mass, or no mass at a.2.

\section{Conservation of matter}

Such heresy clashed with the law of conservation of matter. However, on this question, Ernst Mach wrote (1893): "Investigators have had another struggle on their hands, and by no means an easy one, the struggle with their own preconceived ideas, especially with the idea that philosophy and science must be founded on theology". And "notions of the constancy of the quantity of matter, of the indestructibility of work or energy, conceptions which completely dominate modern physics, all arose under the influence of theological ideas". (See also Gregory, 1969, p.149). Science has not grown from orderly development of fundamentals, but from random empiricisms seen through the constraining filters of our creeds.

\section{Creation}

Creation, and the ultimate origin and destiny of the universe, was also a perennial problem. Postulation of a creator did no more than start the chicken-and-egg infinite series of creators to create creators, or if not an infinite series, the first term would have to be Aristotle's imaginary uncaused cause, unique and different in concept from all the terms which followed.

Here again there was an obvious clue. For just as in the algebra of complex numbers the real terms and the imaginary terms can be segregated as separate complete statements, so with complex propositions involving both material and spiritual these immiscibles should yield segregated propositions each complete in themselves. This could only mean that the sum of the material could only be zero before and after postulated metaphysical excitation.

However, theology aside, ail theories of cosmology require creation, even though creation of matter is contrary to physics' own conservation laws. Bondi (1960, p.9) has crystallized three classes of answer concerning the 'beginning' of the universe: (a) 'The 'beginning' is a singular point on the border of the realm of physical science. Any question which refers to antecedents of the beginning or the nature of the beginning [e.g. creation] can no longer be answered by physics and is not a proper 


\section{S. Warren Carey}

question for it." The laws of physics come into being with creation but do not explain creation. Here physics begs the question of creation by sweeping it under the mat. As McCrea (1964) puts it, creation happened once and for a11, and therefore is not subject to ordinary scientific discussion, but determines the initial conditions for the rest of the discussion.

(b) "There was no 'beginning'. The universe on the large scale is either unchanging or possibly going through cyclical changes, but is of infinite age". Steady-state theories and the 'perfect' cosmological principle belong to this category. Creation is continuous but not explained by physics. The 'arrow of time' has no place. Initial conditions cannot be discussed because the universe always existed, essentially as it is now.

(c) 'The 'beginning' was a particularly simple state, the simplest, the most harmonious and most permanent we can imagine. It contained within itself, though, the seeds of growth and evolution, which at some indefinite moment started off a chain of complicated processes which by now have changed this to our present universe". The laws of physics are universal and have no exclusions nor exceptions whatever. My own philosophy, which I will present to you to-night belongs to this third category, but includes continuous creation compatible with the laws of physics. The beginning is indeed the simplest and most harmonious conceivable--a universal nul1, a cypher, which integrates the cosmos with the infra-nucleon.

UNIVERSAL NULL

Thus severa 1 lines of thought led me to the same answer, that the sum total of the universe is zero. That the sum of all matter and energy of the universe is zero, that the sum of all momentum in the universe is zero, and that the sum of all charge in the universe is zero--a universal null! In such a model all the conservation laws are maintained--at zero. Entropy and temperature mutually cancel at absolute zero as required by the Nernst theorem. Differing exponents are irrelevant, at zero. The universal null model predicts Dirac's large-number equivalences, and solves the prob$1 \mathrm{em}$ of creation (because nothing is really created), and of 0lber's paradox; it accounts for the gross thermodynamic disequilibrium of the universe and its waxing entropy and mean temperature, and gives meaning to the arrow of time.

\section{Momentum}

A single particle in a universal void has neither velocity nor momentum. Once there are two particles, each has velocity and momentum relative to the other. The momentum of the one cancels that of the other. The sum of all momentum of the universe is, I suggest, zero.

\section{Mass-energy}

According to Mach's principle a single particle in a void has zero inertial mass. Infinitesimal force accelerates it. Energy is zero because inertial mass is zero so that potential energy $m g h$ and kinetic energy $\frac{1}{2} m v^{2}$ are each zero. Inertial mass is the effect of all matter in the universe. Einstein also emphasized that a single particle in a void could not have inertia for there can be no inertia of matter against space, only inertia of matter against matter. Energy is a direct first power function of inertial mass. The primitive form is $m g h$, which may convert to any of the other energy modes. Universal potential energy (and from this the derivative energy modes) is directly proportional to total inertial mass of the universe. As mass has grown from the initial nul1, so has energy, pori passu.

Møller (1958) has formulated a consistent expression for the total energy density of the universe consisting of a matter part and a gravitational part. When this expression for the energy density is applied to the case of the metric for a homogeneous 


\section{A Philosophy of the Earth and Universe}

and isotropic universe, "the energy density is zero everywhere and at all times. This means that the positive matter energy is constantly counterbalanced by a corresponding amount of negative gravitational energy."

Energy and mass are obverse and reverse of the same coin, we could call them positive and negative, and write $E=-m e^{2}$, but this would be no more nor less correct than our convention of positive and negative charges, or writing positive and negative for $\mathrm{N}$ and $\mathrm{S}$ poles, left and right, or for clockwise and anticlockwise spins. Just as Mach and Einstein have the potential energy of the entire universe as the direct cause of inertial mass, so the kinetic energy of temperature and radiation would be the direct cause of relativistic mass. No-one really knows what mass, charge, polarity, spin, strangeness are; we simply apply these terms to describe phenomena. All such phenomena occur as gemini, universal twins, which appear together, and mutually cancel each other in the totality of the universe. What is matter? Schatzman (1958) has pointed out that the premise of quantum mechanics is that all elementary particles which make up matter (1eptons, mesons, hyperons) transform into each other, and hence are but different aspects of the same concept. In the etherial times, it was easy to conceive them as strains in the ether, but since the ether has evaporated to a void, we are left with the particles and the strain energy gemini, which mutually cancel to zero.

\section{Dirac's large numbers}

The universal null model not only predicts, but requires the coincidences of the very large dimensionless numbers of physics and cosmology, first recognized by Dirac (1937), and is the only cosmological theory yet to do so. Dirac wrote: "Any two very large dimensionless numbers occurring in Nature are connected by a simple mathematical relation, in which the coefficients are of the order of magnitude unity." Dimensionless numbers come only from comparing paired parameters, for example a mass to a mass, an energy to an energy, a force to a force, a potential to a potential. The table shows some of Dirac's pure numbers from combination of physical constants

\section{Dirac Dimensionless Numbers}

\begin{tabular}{|c|c|c|c|}
\hline$e=$ electronic charge & $\frac{\text { force }}{\text { force }}$ & $\frac{e^{2}}{\gamma m_{\rho} m_{e}}$ & $=0.23 \times 10^{40}$ \\
\hline $\begin{array}{l}m_{e}=\text { mass of electron } \\
m_{\rho}=\text { mass of proton }\end{array}$ & $\frac{\text { length }}{\text { length }}$ & $\frac{c T}{\left(\frac{e^{2}}{m_{e} c^{2}}\right)}$ & $\doteqdot 4 \times 10^{40}$ \\
\hline$\gamma=$ constant of gravitation & & & \\
\hline$c=$ velocity of light & mass & $\underline{\rho_{0} c^{3} T^{3}}$ & $\doteq 10^{80}=\left[10^{40}\right]^{2}$ \\
\hline $\begin{array}{r}\rho_{O}=\text { mean density of matter } \\
\text { in the universe }\end{array}$ & $\overline{\operatorname{mass}}$ & $\overline{m_{\rho}}$ & $\div 100=[10]^{2}$ \\
\hline $\begin{array}{r}T=\text { reciprocal of Hubble's } \\
\text { constant }\end{array}$ & $\frac{\text { energy }}{\text { energy }}$ & $\gamma \rho_{\mathrm{O}} \mathrm{T}^{2}$ & $\doteqdot 1$ \\
\hline
\end{tabular}

of cosmic and microphysics (see Bondi 1960, p.60). The first is the ratio of the electrical to gravitational force between an electron and proton. The second is the ratio of the notional "radius" of the universe to the "effective" radius of an electron. The third is the ratio of the mass of the universe to the mass of a proton (which is approximately the number of atoms in the universe if it were all hydrogen). It is incredible that the concurrence of these and several other large numbers at $10^{\circ}, 10^{40}$, $10^{80}$, should be chance coincidence. It is even more astonishing that the fourth line (which in fact divides the third line by the product of the first two lines, and hence combines a11 the fundamental units listed) should come out at unity. For this is the ratio of the rest energy of any particle to the potential energy of the rest of the 
universe in its field:

$$
\begin{aligned}
& \begin{array}{l}
\text { Potential energy of } \\
\text { universe in field of } m=\frac{m y \times \text { mass of universe }}{\text { radius of universe }}
\end{array} \\
& \sim \frac{m \gamma \rho_{O} c^{3} \mathrm{~T}^{3}}{\mathrm{cT}} \\
& \sim m c^{2} \gamma \rho_{0} T_{2} \\
& \text { Inertial energy of } m=m c^{2}
\end{aligned}
$$

Bondi (Loc. cit.) commented: "The likelihood of coincidences between numbers of the order of $10^{40}$ arising for no reason is so small that it is difficult to resist the conclusion that they represent the expression of a relation between the cosmos and microphysics, a relation which is not understood." But in the null universe the relation is crystal clear, and inevitable. For each new nucleon "created" adds its moity to the universal potential energy, and hence the inertial mass must equal the potential energy of the universe.

\section{The Beginning}

In this simple system, commencing from a universal null, infinitely small perturbations yield small amounts of matter, infinitely dispersed, balanced by infinitely small amounts of potential energy. The matter-energy sum remains zero, however 1 arge or however small the total dispersed mass or the total compensating potential energy, or however much transformation may occur in the form of energy. By analogy, we may compare the gravity-wave perturbation of a placid pond, or, in the business world, the creation of colossal operating credits and assets offset by compensating debits, which together feed enormous industrial activity--with no initial capital beyond a book entry, and a current totality of zero on liquidation.

Indeed, if one conceives a universal void - null time, null matter, null space, should nothing ever happen? Should there not be a random walk between the possible states of zero? Ultimately miniscule random perturbations should set off the compensating plus and minus, up and down, positive and negative, matter and potential, temperature and entropy, so that in due course a universe as we observe it would be the inevitable ultimate result. Even starting from such a universal void, if something could happen, sooner or later it will happen. Entropy of a universal void is zero. Disorder and increasing entropy should inevitably result from the Clausius law that entropy tends towards the maximum.

In this model, the universe develops into a state of perpetual motion which we intuitively exclude from our philosophy. But a virtual perpetual motion is possible for the universe as a whole, for motion itself is part of the definition, and its perpetual sum remains zero. Likewise energy to rum it cancels for it only exists in counterpoint to inertial mass. Normal logic fails in propositions which deal with the validity of their own selves.

Gribbin (1976) has criticised the oscillating universe model of Landsberg and Park (1975), in which successive cycles expand to bigger and bigger radii, by pointing out that running the calculation backwards would regress the oscillation to an "infinitesimal hiccup some large number of cycles ago" -- in other words, this model al so emerges from a universal null. Dirac's initial embryo, Lemâtre's primeval atom, Gamow's ylem, and Gribbin's infinitesmal hiccup were all initial nullities!

\section{Entropy}

The entropy and temperature of the null initial condition should be zero, so that any subsequent condition of the universe would involve increase of entropy, and of mean 


\section{A Philosophy of the Earth and Universe}

temperature. Statistically we could write $S=k T$ (entropy $S$, Boltzman's constant $k$, $T$ absolute temperature) so that entropy is the geminus of thermal energy. As the mean temperature of the universe rises so does entropy to a theoretical maximum at the socalled "heat death" of the universe. Oscillating universe theories meet a paradox with respect to entropy. Le Maitre $(1958, \mathrm{p} .9)$ has pointed out that "the fact that this expansion is made of matter which has already been used would result in its distribution being in a state of maximum entropy". Hence in the new phoenix universe entropy must run backwards -- unless entropy is limited in its field of validity.

\section{Site of matter-energy creation}

Many cosmogony models postulate the continuous creation of matter in the depths of space between the galaxies (e.g. Bondi and Gold, 1948, and Hoyle, 1948), either as hydrogen atoms, or as whole stars, or even as embryo galaxies (Stothers, 1966). Stothers proposes matter is created (as quasars) where it is lacking because it is lacking. McCrea, on the other hand, has proposed (1964) the continual creation of matter as a property of existing matter, and hence predominantly in the nuclei of galaxies. Dirac (1974) debates the relative merits of "multiplicative creation" with matter entering the universe where it is already most concentrated corresponding to Einstein's cylindrical closed universe, and "additive creation" which would satisfy Stother's model where the matter appears in the most tenuous space. The latter corresponds to flat Minowski space originating with the "big bang". In this argument I agree with McCrea and deny Stothers because of the relative energy demands of the two propositions.

To inject one particle in the remote depths of intergalactic space requires a birth endowment for that particle equal to the total potential energy to fall to the centre of mass of the nearest galaxy. Energy endowment to inject a particle at the galactic centre of mass is virtually zero (not quite zero because every other particle receives a trivial increment in its potential energy to fall thither). In a mature universe, most matter would enter at the centres of nebulae - as indeed Jeans deduced empirically (1928): "The type of conjecture which presents itself somewhat consistently, is that the centres of nebulae are of the nature of singular points at which matter is poured into our universe from some other and entirely extraneous spatial dimension, so that to a denizen of our universe they appear as points at which matter is being continuously created." What is this extraneous spatial dimension? Energy of course, the geminus of mass, the potential energy of the universe. To extend Jeans and McCrea further, it would seem plausible that all matter grows at a rate which is a power function of its own concentration.

Bondi (1960) suggested that the mean density of intergalactic hydrogen could be as high as $10^{-25} \mathrm{~g} / \mathrm{cm}^{3}$ or $10^{5}$ times the mean density of the galaxies if their matter was uniformly dispersed through space $\left(10^{-30} \mathrm{~g} / \mathrm{cm}^{3}\right)$. In Bondi's model by far the predominant part of all matter is in the depths of space. There is a sharp contrast between the Jeans-McCrea mode1 of a nebula (which I support) and the Bondi-Stothers mode1; in ours matter enters at the centres of nebulae and disperses outwards to the limits of the nebula; in theirs the matter enters in the depths of space and condenses into the nebulae. The problem of angular momentum distribution seems to deny this latter proposition, a problem which evaporates in the Jeans-McCrea model. Thus both the potential energy endowment and the angular momentum distribution indicate creation of matter where it is concentrated, not where it is absent. Furthermore contracting galaxies in an expconding universe lack philosophical elegance. In our model, everything expands, Earth, the Earth-Moon system, the Solar system, the galaxies, the whole universe, and what is astonishingly relevant, the Hubble constant measures each of these independent expansions, as McDougali and his co-authors pointed out (1963).

Cosmic Repulsion

A11 of Newton's empirical laws - of universal gravitation, of accelerated motion, 


\section{S. Warren Carey}

of viscous flow, and Hooke's empirical law of the proportionality of stress and strain, are probably invalid if pursued far beyond the range of the empiricism. The relativistic mass of kinetic energy is always present even at low velocities, but is much below the threshold of experimental detection, although this term dominates at speeds approaching the velocity of light. Viscous and plastic terms are always present during the elasticity experiments but are too small to be detected in short duration experiments with the materials used, although they dominate when experiments are sustained for times far exceeding the Maxwell relaxation time. So-called non-Newtonian flow is present in all viscous experiments, but in low viscosity fluids is below the threshold of measurement. However, the distinction between fluids with Newtonian viscosity and other substances regarded as non-Newtonian is solely a matter of scale and refinements of the measurements.

Likewise I doubt whether Newton's law of Universal gravitation

$$
F=G \frac{m_{1} m_{2}}{d^{2}} \text {.............. . }
$$

is valid for cosmic distances, and I suggest that an additional term is always present even though too small to be detected in less than cosmic scale. Such a term might take a form like:

$$
F=G m_{1} m_{2}\left(\frac{1}{d^{2}}-\frac{a d^{2} H^{4}}{c^{4}}\right) \ldots . . . . . . .
$$

where $H$ is Hubble's constant and $c$ the velocity of light and $a$ is a scaling constant. $H / C$ is a very small quantity approaching zero, so for small (e.g. local and astronomic) values of $d$ this relation reverts to Newton's law of gravitation for all practical purposes, but for cosmic values of $d, 1 / d^{2}$ approaches zero and the second term becomes significant but negative, replacing gravitational attraction by cosmic repulsion. A graph of $F$ against $d$ would have a form such as:

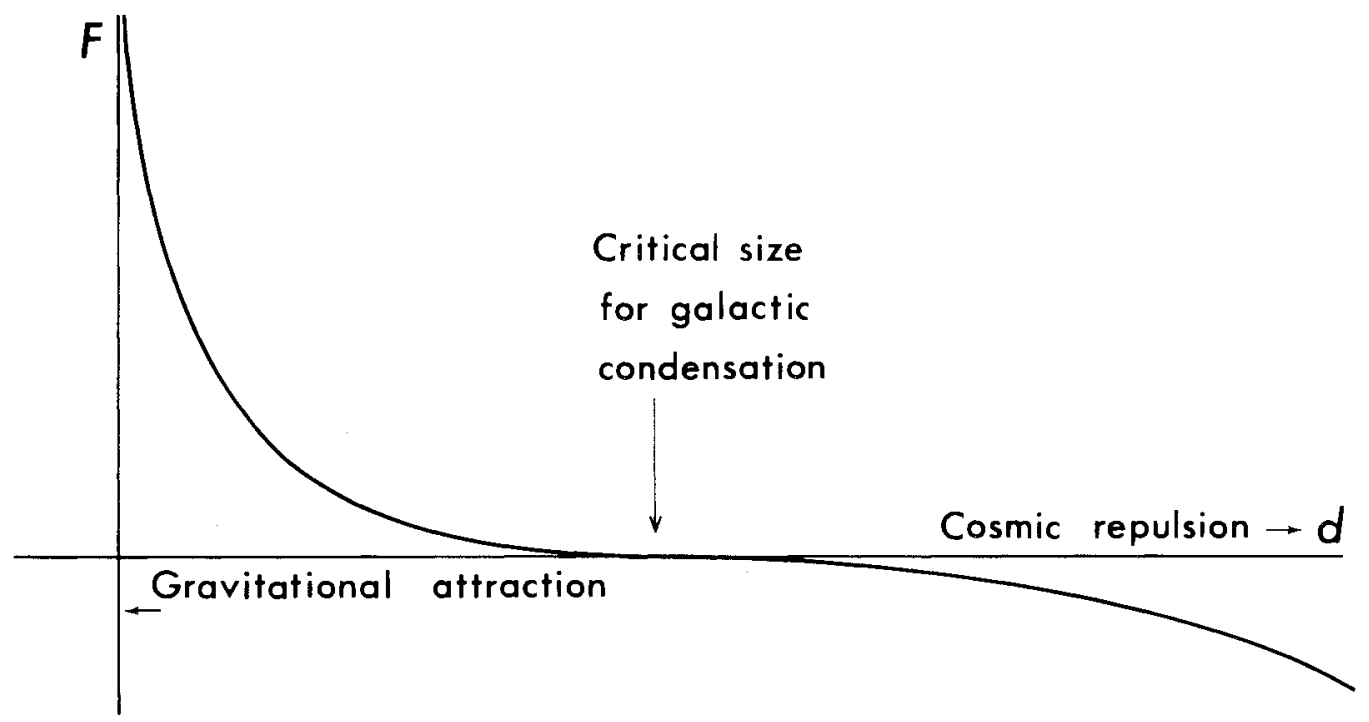

If Newton's law of gravitation (as expressed by (1) above) applied universally, the whole universe should have collapsed under gravity. If however the law took some form like (2) above then gravitational condensation would occur up to inter-galactic distances, but at cosmic distances repulsion would supersede. 
Anti-Matter

"Anti-matter", so-called, exists here in our environment. A positron is the "anti-particle" of the electron. But the positron is "anti" to the electron in little more than charge. Its mass and energy are measured by the gram and erg, whereas a genuinely "anti" electron would measure its mass by the marg, its energy by the gre, and its arrow of time would dart backwards. Perhaps such an anti-universe exists with mirror symmetry with ours on the other side of $\mathrm{T}_{\mathrm{o}}$ somewhere beyond infinity. But if so we would never be aware of it because it could never meet up with any point of our universe in the coordinate system of $x, y, z$, iT.

\section{Universal Rotation}

One of the most striking facts of the universe is that everything in it rotates-atoms, planetary systems, and nebulae. Why should this be so? What is implied? Every hydrogen atom has spin. For example, in a proton-precession magnetometer the spins of al1 these protons in a bottle of water are axially aligned by a solenoid around the bottle and when this coil current is switched off each spinning proton suffers the torque of the local magnetic field, which makes it precess at a rate dependent on the strength of that field. It has generally been assumed that spin axes of matter are normally oriented randomly. But if matter comes into being as the twin of potential energy (perhaps as sub-atomic particles) any ambient field from the body would polarize the nascent spins to the counter sense. Hence, with each particle born, the particle and the host body would receive equal and opposite angular momenta. If we assume the sun to be wholly hydrogen and add all the momenta of its constituent protons, and compare this sum with the angular momentum of the sun, we fall short by some orders.

Moreover, the angular momentum of the sun (which carries most of the matter) is only one fiftieth of the angular momentum of the solar system, so clearly the assumptions of the calculation cannot be the whole answer. Nevertheless, I suspect that it is in this direction that the enigma of universal rotation should be sought.

Notwithstanding this suggestion, universal rotation may derive from fundamental cosmic properties seeing that Hubble's constant has the dimensions of $1 / \mathrm{T}$ which is the same as the dimensions of angular velocity. There is however a vector difference: as we move out along the radius of a rotating body, linear velocity normal to the radius increases directly in proportion to the radius. The same is true with increasing cosmic distances except that the waxing velocity is along the radius not normal to it. Are rotation and Hubble dispersion related as are magnetic and electric vectors?

Standard theory assumes that the Hubble velocity increase is constant, i.e. that it is directly proportional to distance. However, this cannot be true. For, as pointed out by others the Hubble constant gives the observed present rate not only of the cosmos, but also of the Earth-Moon system, and of Earth expansion. However, in each case linear retrogression from the present gives obviously false rates. The whole cosmos would be concentrated in a small space 1.9 aeons ago, Moon would be back here at about the same time, and Earth would have reduced to a very small body - all this within the middle of the Proterozoic. Clearly Hubble's "constant" must be timedependent and regress asymptotically to $\mathrm{T}_{\mathrm{o}}$.

The Solar System as $G$ changes

Application to the solar system of the continuous creation of matter and of the change in $G$ with time implies changes in the diameters of the planetary orbits as well as variation in the luminosity of the sun, which should show up in the geological record. Chin and Stothers (1975) have investigated this question in the case of multiplicative creation (that is where mass grows according to its own concentration) and they found "the rather surprising result that [these solar models] are nearly the same as the final model based on standard theory! This occurs in spite of the widely disparate initial masses, occasioned by the use of a full range of choices for to. The reason for this similarity is that the effect of a larger $G$ in the past is to 
increase the luminosity, whereas lower stellar mass decreases it." They go on to investigate the effects on the orbits, and concluded that the deduced temperatures at the surface of the earth do not conflict with palaeogeographical evidence.

Do rocks grow?

Chin and Stothers however then ask: "But why are we11-preserved Precambrian and early Cambrian fossils in essentially perfect shape if their masses have increased by a significant percentage?" This enigma arises from their assumption (following Dirac) that in multiplicative creation all atoms would multiply kind for kind - silicon for silicon and so on. However surely this would be in the highest degree improbable. The likely kind of multiplicative creation would be in the simplest state possible "infra-quarks" or whatever. In due course the first atoms would be the simplest atomic hydrogen, which would diffuse out of the rock either as gas or as water. Every volume of sedimentary rock in a geosyncline suffers transit through it of several times its own volume of fluids, mainly water but also hydrocarbons as gases or liquids, yet no distortion of fossils results. In fact the great majority of fossils do show large degrees of strain, but not from such passage of fluids, but from ordinary compaction under load and from tectonic deformation of the rock.

Towe (1975) has claimed that "if atomic distances vary as $t^{-1}$ as required by Dirac's large-numbers theory, then the d-spacings in crystal lattices in geologically older minerals should be observedly different from those in the same minerals forming today. The geological evidence, however is strongly against such a prediction: the lattice dimensions of a $3 \times 10^{9}$ year-old quartz crystal are the same as the lattice dimensions of quartz grown in the laboratory." Of course they are, and would be under Dirac's theory. Quartz would have grown initially with the $d$-spacings appropriate to the time of origin. But as the ambient fields and "constants" change, so would the size of the unit cell whatever its juvenility or senility. Gittus (1975) and Towe (Zoc. cit.) make the same false assumption as do Chin and Stothers above, that silicon would replicate as silicon and so on.

In any case cosmic empiricism suggests that growth of matter is a function of its own concentration, and geological empiricism suggests that most growth takes place in the core, and perhaps to a less degree in the mantle. The controlling function may involve density of matter and perhaps pressure and temperature.

\section{Earth's Surface gravity}

Stewart (1977) has summarized five sets of data which put limits on the former magnitude of gravity at the earth's surface $(g)$ compared to the present magnitude $\left(g_{0}\right)$ : "1. The permanent existence of a terrestrial atmosphere over the last $3.7 \times 10^{9}$ years $\left(0.33 g_{0}<g\right) .2$. Dimensions of vertebrates: Jurassic Apatoscurus $\left(g<1.2 g_{0}\right)$ and Upper Cretaceous Pteranodon $\left(g<2 g_{0}\right)$. 3. Miocene overconsolidation of the London Clay $\left(0.5 g_{0}<g \leqslant g_{0}\right)$. 4. Phanerozoic upper mantle mineralogy $\left(0.4 g_{0}<g<2.2 g_{0}\right)$. 5. Crustal mineralogy during the Phanerozoic $\left(0.5 g_{0}<g<1.4 g_{0}\right)$ and Precambrian $\left(0.5 g_{0}<g<1.9 g_{0}\right)$." Stewart goes on to state that "These limits can be used to put constraints on hypotheses involving Earth expansion and changes in the universal constant of gravitation." This corollary is only true if the further assumption is made that the matter and mass of the earth have been essentially constant. For my part I am convinced that this assumption is false, and on a cosmic scale leads to the absurdity of Le Maltre's ultra-embryo already discussed.

\section{Are we alone?}

A decade ago I commented in another oration (Carey 1967) that one of the puzzles of the universe is why we have not yet recognized signals from other thinking beings. We are flooded with diverse radiation from all over the galaxy and beyond, but have found no sign of wilful modulation. Surely, with so many millions of suns comparable to ours, it is highly improbable that we are the only cognitive beings, or the most 
advanced. Why have we not heard from them?

One answer is that they would not have seen any evidence of us--yet. Or is eventual self-destruction inherent in any competitive evolutionary system soon after it achieves the technical competence to do so, so that there never has been, nor ever will be, a race much more advanced than ours? Then, again, perhaps we are not searching the right medium. A century ago we were quite unaware of radio waves. Perhaps when we learn to modulate and demodulate coherent light efficiently, we may find all the messages we seek. After a11, a 1ight channel can carry vastly more information than any radio channel, and may well be the preferred medium for those who know how to use it. Shall we sudden $1 y$ break in on a vast communication system of the galaxy? Perhaps we have to leam to communicate by still some other carrier as yet unrecognized.

\section{The Arrow of Time}

The cosmological principle is the analogue in cosmology of the principle of uniformitarianism in geology. In its "perfect" form it postulates that the laws of physics are invariant through space-time, that a sample of the universe of sufficient size does not differ significantly from any other sample elsewhere in space or at some other time. The perfect cosmological principle (which is the geologists' principle of uniformitarianism without the geologists' 1 aw of superposition) supports steady-state theories, but not evolutionary theories, nor increasing entropy, nor the arrow of time.

For my part, empiricism does not support the steady state in respect of time. Pochoda and Schwarzchild (1964) found that steady-state additive models predicted solar neutrino ratios several orders too high, whereas Chin and Stothers (1975) showed that multiplicative creation (with matter increasing in proportion to its own concentration) predicted a solar neutrino flux consistent with standard solar models. Moreover, neither in local astronomy nor in gross cosmology is now the same as then. For example, if we reverse the observed acceleration of the moon, the moon would be back at the earth during the Proterozoic Era. Similarly if we reverse the recession of the galaxies they would herd in a small space a few aeons ago. The oldest rocks known on Earth or Moon are between three and four aeons old and meteorites have similar age. $\mathrm{T}_{\mathrm{o}}$ for all seems to be a few aeons ago. These regressions are probably too simple and perhaps should be exponential, which would extend $\mathrm{T}_{0}$ asympotically towards infinity. But neither the Earth-Moon system nor the whole cosmos appear to be invariant with time, as would be required by the perfect cosmological principle.

The perfect cosmological principle and its steady-state corollary seem to have another fatal objection arising from the gross thermodynamic disequilibrium of the universe. Olber, early last century, found that in an equilibrium system each body would eventually radiate as much energy as it received, and universal light would saturate the sky. This clearly was not so--hence Olber's paradox of stars in a black sky. In a steady-state universe without zero time thermodynamic equilibriun should have been attained. Truly we might postulate a dynamic equilibrium, but such a system would be inherently unstable, and could hardly represent a steady-state universe. Escape from this dilemma could be conceived in an expanding universe where all matter approached the seeable boundary of the universe at the velocity of light before thermodynamic equilibrium could be attained. It is true that the red-shift implies an energy sink, and that as the recession velocity approaches the velocity of light, the radiant energy back along its path approaches zero. However there would be an infinity of such receding galaxies, and the energy return would be the product of tending-towardzero and tending-toward-infinity!

The problem of zero time, and of mirror symetry across it, evaporates in the null model, because zero time must then be pursued back towards the initial infinity, before the appearance of a random perturbation. The arrow of time has not deflected since this infinity, hence the empiricism of our intuition. 
Creation and The Spiritual

Creation may be considered in two distinct ways - as a question for physics or for theology. Even though these may become entwined in conceptual discussion, I stated earlier that they can be separated just as the real and imaginary terms in a complex mathematical statement can be separated into two discrete statements, one containing all the real terms and the other containing all the imaginary terms.

People differ on the validity of a spiritual world. I have no personal experience of anything spiritual so I can neither affirm nor deny the validity of a spiritual domain. However quite a number of my close acquaintances positively affirm such experience. I am satisfied that they are truthful witnesses, though this does not exclude the possibility of their self-deception.

If then we do not exclude the spiritual, nevertheless it can be entirely separated from the physical as in the foregoing mathematical analogue. No recorded mass, charge, magnetic field, or electromagnetic radiation has been attributed to a soul. Heaven and hell have no physical location in the mantle, on the moon, or in interplanetary, or interstellar space. All of us have had the clearest and most detailed visual inages in dreams or other states of trance, but no light waves are associated with them, and from my own experience the time scale of sequential events can be compressed by several orders.

It is true also that the solution to difficult problems may become obvious in our disturbed sleep, but I do not regard this as external inspiration. Rather, during our dreaming sleep the brain churns over the waking problem, but with an important difference: it is no longer restricted by the constraints of our relevant prejudices. So often it is what we think we know which blinds us to the truth, and to the solution to our problem.

Thus none of the phenomena of dreams, or visions, or inspiration, known to me demand explanations beyond natural science. Creation itself is no longer a problem because in the null universe nothing - not mass, not charge, not energy - is created. The material universe is integral without intervention of the spiritual. But this does not deny or exclude the possibility of other phenomena.

\section{CONCLUSION}

Commencing with a universal void, the inseparable twins of positive and negative charge, of north and south polarity, of momentum vectors and cancelling momentum vectors, of rotation and counter rotation, of inertial mass-energy and gravitational potential energy, gravitational attraction and cosmological repulsion, have waxed wider with time, with increasing entropy and mean temperature, but always reduce to zero in the ultimate reckoning. For how else could the immensity of the universe come into being?

When the Indians invented zero to make a continuous series of numbers from minus infinity to plus infinity the concept was so obvious that we marvel that it had not been thought of before. So also the universal null emerges with obvious simplicity, as inevitable.

\section{POSTSCRIPT}

Since delivering this address I have found that Edward P. Tryon had published in Nature of December 14, 1973 a universal nuIl hypothesis under the title, "Is the universe a vacuum fluctuation?" Tryon concluded that the universe did appear from nowhere about $10^{10}$ years ago, and to be consistent with the laws of physics the net value of all conserved quantities must be zero. His universe is homogeneous, isotropic, 
and closed, and consists equally of matter and anti-matter. Tryon a1so pointed out that inertial mass and gravitational potential energy are intrinsically equivalent and mutually cancelling. He also quotes Bergmann's argument that a closed universe must have zero total energy, because it would be topologically impossible for any gravitational flux lines to go outside the limits of the universe, and hence the sum of the energy of the whole universe must be zero.

\section{REFERENCES}

Bondi, H., 1960: COSMOLOGY. 2nd edn, Cambridge U. Press. 182p. and Gold, T., 1948: The steady-state theory of the expanding universe. Roy. Astron. Soc., Mon. Not., 108, 252-270.

Carey, S.W., 1967: 2000 AD - Prognosis. Med. Joum. Aust., 25, 1235-1242

(Mills Memorial Oration to Roy. Aust. Coll. Physicians) 1976: THE EXPANDING EAR'Tl. Elsevier, Amsterdam, 1-488.

Chin, C.W. and Stothers, R., 1975: Solar test of Dirac's large numbers hypothesis. Nature, 254, 206-207.

Dirac, P.A.M., 1937: The cosmological constants. Nature, 139, 323.

, 1974: Cosmological models and the large numbers hypothesis. Roy. Soc. Lond. Proe., A338, 439-446.

Gamow, G., 1952: THE CREATION OF THE UNIVERSE. Viking Press, New York, 147p.

Gilbert, C., 1956: Dirac's cosmology and the general theory of relativity. Roy. Astr. Soc., Mon. Not., 116, 684-690.

Gittus, J.H., 1975: Dirac's large numbers theory and the structure of rocks. Roy. Soc. Lond., Proc., A343, 155-158.

Gregory, M.S., 1969: AN INTRODUCTION TO EXTREMUM PRINCIPLES. Butterworths, London. 1-196.

Gribbin, J., 1976: Oscillating universe bounces back. Nature, 259, 15-16.

Hoyle, F., 1948: A new model for the expanding universe. Roy. Astron. Soc., Mon. Hot., $108,372-382$.

Jeans, J.H., 1928: ASTRONOMY AND COSMOGONY. Cambridge U.P.

Klepp, H.B., 1964: Terrestrial interplanetary and universal expansion. Nature, $201(4920), 693$.

Landsberg, P.T. and Park, D., 1975: Entropy in an oscillating universe. Roy. Soc. Lond., Proc., A346, 485-495.

Lemaître, G., 1951: THE PRIMEVAL ATOM. Van Nostrand, New York. 186p. , 1958: The primaeval atom hypothesis and the problem of the clusters of galaxies. 11th Solvay Conf., Brusse1s, 1-25.

MacDouga11, J., Butler, R., Kronberg, P., and Sandqvist, A., 1963: Astronomy: A comparison of terrestrial and universal expansion. Nature, 199, 1080.

McElhinny, M.W., Haile, N.S., and Crawford, A.R., 1974: Palaeomagnetic evidence shows Malay Peninsula was not a part of Gondwanaland. Nature, 252 (5485), 641-645.

Mach, E., 1893: DIE MECHANIK IN IHRER ENTWICKLUNG HISTORISCH-KRITISCH DARGESTELLT Trans: T.J. McCormack, 1960: THE SCIENCE OF MECHANICS: A CRITICAL AND HISTORICAL ACCOUNT OF ITS DEVELOPMENT. Open Court Publ. Co., La Salle, I1linois. 1-634.

McCrea, W.H., 1964: Continual creation. Roy. Astron. Soc., Mon. Not., 128, 335.

Milne, E.A., 1935: RELATIVITY, GRAVITATION AND WORLD STRUCTURE. Oxford U. Press.

Mф11er, C., 1958: Discussion of Hoyle, "The steady state theory". 11th Solvay Conf., Brussels, p.74.

Pochoda, P., and Schwarzchild, M., 1964: Variation of the gravitational constant and the evolution of the sun. Astrophys. Joum., 139, 587-593.

Schatzman, E., 1958: Discussion of Hoyle, "The steady state theory". 11th Sozvay Conf., Brussels, p.75.

Stewart, A.D., 1977: Quantitative limits to palaeogravity. Quart. J. geol. Soc. Lond., 133, 281-291.

Stothers, R., 1966: Quasars as the origin of primordial matter in a steady-state universe. Roy. Astron. Soe., Honth. Not., 132, 217-223. 


\section{S. Warren Carey}

Towe, K.M., 1975: Crystal structures, the earth and Dirac's large numbers hypothesis. Nature, 257 (5522), 115-116.

Walker, A.G., 1936: On Milne's theory of world-structure. London Math. Soc., 42, $90-127$. 